\title{
Non-contiguous finished genome sequence of plant- growth promoting Serratia proteamaculans S4
}

\author{
Saraswoti Neupane ${ }^{1}$, Lynne A. Goodwin², Nils Högberg Nikos C. Kyrpides ${ }^{3}$, Sadhna \\ Alström¹ , David Bruce ${ }^{2}$, Beverly Quintana ${ }^{2}$, Christine Munk², Hajnalka Daligault' ${ }^{2}$, Hazuki \\ Teshima $^{2}$, Karen Davenport ${ }^{2}$, Krista Reitenga ${ }^{2}$, Lance Green ${ }^{2}$, Patrick Chain ${ }^{2}$, Tracy Erkkila ${ }^{2}$, \\ Wei Gư ${ }^{2}$, Xiaojing Zhang ${ }^{2}$, Yan Xu², Yulia Kunde², Olga Chertkov², James Han³, Cliff Han², \\ John C. Detter ${ }^{2}$, Natalia Ivanova ${ }^{3}$, Amrita Pati ${ }^{3}$, Amy Chen $^{3}$, Ernest Szeto ${ }^{3}$, Kostas \\ Mavromatis ${ }^{3}$, Marcel Huntemann ${ }^{3}$, Matt Nolan $^{3}$, Sam Pitluck $^{3}$, Shweta Deshpande ${ }^{3}$, Victor \\ Markowitz ${ }^{3}$, Ioanna Pagani ${ }^{3}$, Hans-Peter Klenk ${ }^{4}$, Tanja Woyke ${ }^{3}$ and Roger D. Finlay ${ }^{1}$ \\ ${ }^{1}$ Uppsala BioCenter, Department of Forest Mycology and Plant Pathology, Swedish Univer- \\ sity of Agricultural Sciences, Uppsala, Sweden \\ ${ }^{2}$ Los Alamos National Laboratory, Bioscience Division, Los Alamos, New Mexico, USA, \\ ${ }^{3}$ DOE Joint Genome Institute, Walnut Creek, California, USA, \\ ${ }^{4}$ Leibniz Institute DSMZ - German Collection of Microorganisms and Cell Cultures, \\ Braunschweig, Germany
}

Correspondence: Saraswoti Neupane (Saraswoti.Neupane@slu.se)

Keywords: Facultative aerobe, gram-negative, motile, non-sporulating, mesophilic, chemoorganotrophic, agriculture

Serratia proteamaculans S4 (previously Serratia sp. S4), isolated from the rhizosphere of wild Equisetum sp., has the ability to stimulate plant growth and to suppress the growth of several soil-borne fungal pathogens of economically important crops. Here we present the noncontiguous, finished genome sequence of $S$. proteamaculans S4, which consists of a 5,324,944 bp circular chromosome and a 129,797 bp circular plasmid. The chromosome contains 5,008 predicted genes while the plasmid comprises 134 predicted genes. In total, 4,993 genes are assigned as protein-coding genes. The genome consists of 22 rRNA genes, 82 tRNA genes and 58 pseudogenes. This genome is a part of the project "Genomics of four rapeseed plant growth-promoting bacteria with antagonistic effect on plant pathogens" awarded through the 2010 DOE-JGI's Community Sequencing Program.

\section{Introduction}

The genus Serratia is a diverse and widely dispersed group of Gammaproteobacteria [1,2]. Some of these have beneficial effects on ecologically and economically important plants [3-4] and others are known as opportunistic pathogens of humans and other organisms [1]. Plant-associated Serratia spp. are of considerable agricultural interest and several strains of $S$. plymuthica have recently been studied in relation to their possible use as biocontrol agents in agriculture [3-4].

Serratia proteamaculans S4 (previously Serratia $s p$. S4) was isolated from the rhizosphere of naturally growing Equisetum plants in 1980 from Uppsala, Sweden. The bacterium is able to enhance the growth of rapeseed plants and inhibit the growth of different fungal pathogens such as Verticillium dahliae, and Rhizoctonia solani. Sequencing the $S$. proteamaculans $\mathbf{S} 4$ genome will therefore assist in the identification of genetic traits underlying its potential and its beneficial effects on plant growth. Here we present the non-contiguous finished genome sequence of S. proteamaculans S4.

\section{Classification and features}

A representative 16S rRNA gene sequence of $S$. proteamaculans $\mathrm{S} 4$ was subjected to comparison with the most recently released databases in GenBank. The NCBI BLAST [5] tool was used under the default settings (i.e. by considering only the high-scoring segment pairs (HSP's) from the best 250 hits). The most frequently matching genus was Serratia (almost 50\% of total matches). When considering high score, coverage and identity - S. proteamaculans 568 was the first match with $100 \%$ identity and $100 \%$ coverage. Other Serratia species with maximum identity were other S. proteamaculans strains $(10 \%)$ with maxi- 
mum identity 99\%, S. fonticola (2\%) with maximum identity 98\%, S. grimesii (3.2\%) with maximum identity 99\%, S. liquefaciens (4.4\%) with maximum identity 99\%, S. plymuthica (3.2\%) maximum identity $98-99 \%$ and unclassified Serratia sp. (22\%) with maximum identity 9899\%. Remaining matches were with Rahnella sp. (2\%) with maximum identity $98-99 \%$ and other uncultured bacterial clones (40\%) with maximum identity $98-99 \%$.

Figure 1 shows the phylogenetic proximity of $S$. proteamaculans S4 to S. proteamaculans 568 (CP000826) as well as its distinct separation from other members of the Enterobacteriaceae. Its phylogenetic relationship was further confirmed by digital DNA-DNA hybridization [10] values above $70 \%$ with the genome sequence of the $S$. proteamaculans 568 using the GGDC web-server [11].

Serratia proteamaculans S4, a Gram-negative, rod shaped, non-sporulating and motile bacterium measuring 1-2 $\mu \mathrm{m}$ in length and 0.5-0.7 $\mu \mathrm{m}$ in width [Figure 2], was isolated from Equisetum roots. The bacterium is a pale yellow colored, facultative aerobe and easily grows on a broad spec- trum of organic compounds including carbon sources such as glucose, sucrose, succinate, mannitol, inositol, sorbitol, arabinose, trehalose, and melibiose. The optimal temperature for its growth is $28^{\circ} \mathrm{C}$ and it can grow in the $\mathrm{pH}$ range $4-$ 10 [Table 1].

\section{Genome sequencing information}

Serratia proteamaculans S4 was selected for sequencing because of its biological control potential and plant growth enhancing activity in rapeseed crops. The genome sequence is deposited in the Genomes On Line Databases [9]. Sequencing, finishing and annotation were performed by the DOE Joint Genome Institute (JGI). A summary of the project information is shown in Table 2 together with associated MIGS identifiers [12].

\section{Growth conditions and DNA isolation}

Serratia proteamaculans S4 was grown on Luria Broth (LB) medium for 12 hours at $28{ }^{\circ} \mathrm{C}$. The DNA was extracted from the cells by using a standard CTAB protocol for bacterial genomic DNA isolation, which is available at JGI [26].

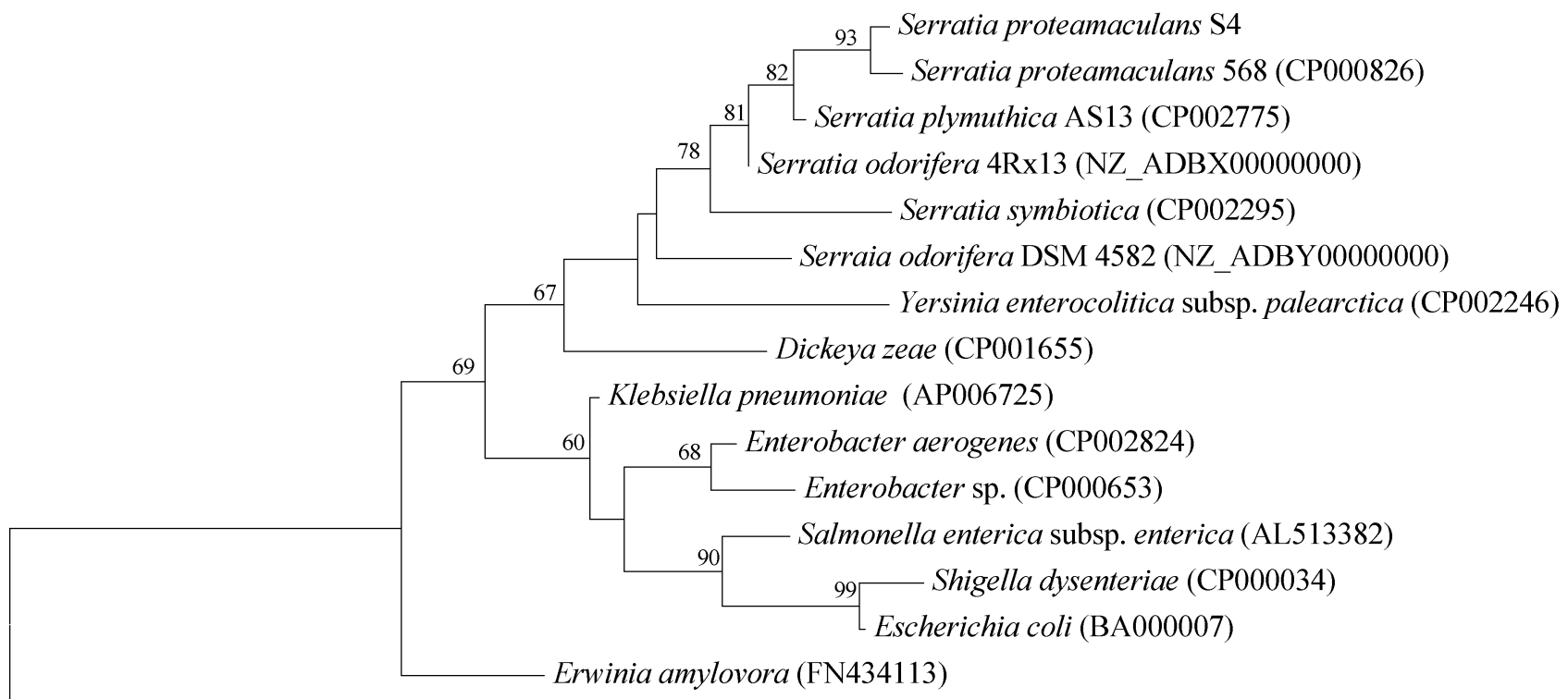

Azotobacter vinelandii (CP001157)

0.02

Figure 1. Phylogenetic tree highlighting the position of $S$. proteamaculans $S 4$ in relation to other type and non-type strains within the family Enterobacteriaceae. The tree is based on 1,489 characters of the 16S rRNA gene sequence aligned in ClustalW2 [6] under the default settings. The tree was constructed using MEGA5 software [7] under the Maximum likelihood criterion and the tree was rooted with Azotobacter vinelandii (a member of the family Pseudomonadaceae). The branches are scaled according to the expected number of substitutions per site. The numbers above the branches are support values from 1,000 bootstrap replicates if larger than $60 \%$ [8]. All lineages with genome sequences are registered in GOLD [9]. 


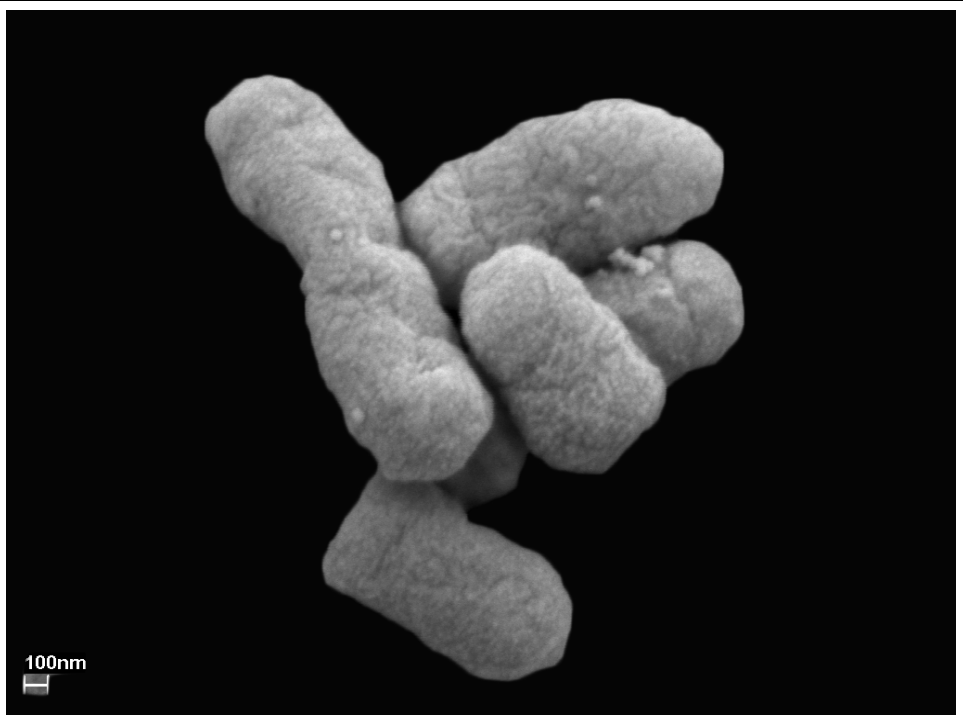

Figure 2. Scanning electron micrograph of S. proteamaculans S4

Table 1. Classification and general features of S. proteamaculans S4 according to the MIGS recommendations [12]

\begin{tabular}{|c|c|c|c|}
\hline MIGS ID & Property & Term & Evidence code $^{\mathrm{a}}$ \\
\hline & \multirow{8}{*}{ Current classification } & Domain Bacteria & TAS [13] \\
\hline & & Phylum Proteobacteria & TAS [14] \\
\hline & & Class Gammaproteobacteria & TAS $[15,16]$ \\
\hline & & Order Enterobacteriales & TAS [17] \\
\hline & & Family Enterobacteriaceae & TAS [18-20] \\
\hline & & Genus Serratia & TAS $[18,21,22]$ \\
\hline & & Species Serratia proteamaculans & TAS $[18,23]$ \\
\hline & & Strain S4 & IDA \\
\hline & Gram stain & Negative & IDA \\
\hline & Cell shape & Rod & IDA \\
\hline & Motility & Motile & IDA \\
\hline & Sporulation & Non-sporulating & IDA \\
\hline & Temperature range & $4-40^{\circ} \mathrm{C}$ & IDA \\
\hline & Optimum temperature & $28^{\circ} \mathrm{C}$ & IDA \\
\hline & Carbon source & $\begin{array}{l}\text { Glucose, sucrose, succinate, mannitol, arabinose, } \\
\text { sorbitol, inositol }\end{array}$ & IDA \\
\hline & Energy source & Chemoorganotrophic & IDA \\
\hline MIGS-6 & Habitat & Wild Equisetum rhizosphere & IDA \\
\hline MIGS-6.3 & Salinity & Medium & IDA \\
\hline MIGS-22 & Oxygen & Facultative & IDA \\
\hline MIGS-15 & Biotic relationship & Plant associated & IDA \\
\hline \multirow[t]{2}{*}{ MIGS-14 } & Pathogenicity & None & NAS \\
\hline & Biosafety level & 1 & TAS [24] \\
\hline MIGS-4 & Geographic location & Uppsala, Sweden & NAS \\
\hline MIGS-5 & Sample collection time & 1980 & NAS \\
\hline MIGS-4.1 & Latitude - & 59 & NAS \\
\hline MIGS-4.2 & Longitude & 17 & NAS \\
\hline MIGS-4.3 & Depth & $0.1 \mathrm{~m}$ & NAS \\
\hline MIGS-4.4 & Altitude & $58-63 m$ & NAS \\
\hline
\end{tabular}

a) Evidence codes - IDA: Inferred from Direct Assay; TAS: Traceable Author Statement (i.e., a direct report exists in the literature); NAS: Non-traceable Author Statement (i.e., not directly observed for the living, isolated sample, but based on a generally accepted property for the species, or anecdotal evidence). These evidence codes are from the Gene Ontology project [25]. If the evidence code is IDA, then the property should have been directly observed, for the purpose of this specific publication, for a live isolate by one of the authors, or an expert or reputable institution mentioned in the acknowledgements. 


\begin{tabular}{lll}
\hline \multicolumn{2}{l}{ Table 2. Genome sequencing project information } \\
\hline MIGS ID & Property & Term \\
\hline MIGS-31 & Finishing quality & Non-contiguous Finished \\
MIGS-28 & Libraries used & $\begin{array}{l}\text { Three libraries: one 454 standard library, one paired end 454 library (10 kb } \\
\text { insert size) and one Illumina library }\end{array}$ \\
MIGS-29 & Sequencing platforms & Illumina GAii, 454 GS FLX Titanium \\
MIGS-31.2 & Fold coverage & $767.4 \times$ Illumina, 8.7 × pyrosequencing \\
MIGS-30 & Assemblers & Velvet version 1.1.05, Newbler version 2.6, phrap version SPS - 4.24 \\
MIGS-32 & Gene calling method & Prodigal (1.4), GenePRIMP \\
& NCBI project ID & 61833 \\
& NCBI taxon ID & 768491 \\
& IMG object ID & 2508501071 \\
& GOLD ID & Gi08429 \\
& Project relevance & Biocontrol, Agriculture \\
\hline
\end{tabular}

\section{Genome sequencing and assembly}

The draft genome of $S$. proteamaculans S4 was generated using a combination of Illumina and 454 sequencing platforms. The details of library construction and sequencing are available at the JGI [26]. The sequence data generated from Illumina GAii $(4,232 \mathrm{Mb})$ were assembled with Velvet [27] and the consensus sequence was computationally shredded into $1.5 \mathrm{~kb}$ overlapping fake reads. The sequencing data generated from 454 pyrosequencing (89.5 Mb) were assembled with Newbler and consensus sequences were computationally shredded into $2 \mathrm{~kb}$ overlapping fake reads. The initial draft assembly contained 50 contigs in 2 scaffolds. The 454 Newbler consensus reads, the Illumina Velvet consensus reads and the read pairs in the 454 paired end library were integrated using parallel Phrap [28,29]. The software, Consed [30] was used for the subsequent finishing process. The software Polisher [31] was used to correct the base errors and increase the consensus quality. Possible mis-assemblies were corrected with gapResolution ([26], unpublished), Dupfinisher [32] or by sequencing cloned bridging PCR fragments with subcloning. The gaps between contigs were closed by editing in the software Consed [30], by PCR and by Bubble PCR (J.-F. Chang, unpublished) primer walks. A total of 95 additional reactions was necessary to close gaps and to raise the quality of the finished sequence. The final assembly is based on $47 \mathrm{Mb}$ of $454 \mathrm{draft}$ data which provides an average $8.7 \times$ coverage of the genome and 4,143.8 Mb of Illumina draft data, which provides an average $767.4 \times$ coverage of the genome.

\section{Genome annotation}

The S. proteamaculans S4 genes were identified using Prodigal [33] as part of the DOE-JGI annotation pipeline [34] followed by a round of manual curation using the JGI GenePRIMP pipeline [35]. The predicted CDSs were translated and used to search the National Center for Biotechnology Information (NCBI) non-redundant database, UniProt, TIGRFam, Pfam, PRIAM, KEGG, COG, and InterPro databases. These data sources were combined to assert a product description for each predicted protein. Non-coding genes and miscellaneous features were predicted using tRNAscanSE [36], RNAmmer [37], Rfam [38], TMHMM [39], and signalP [40]. Additional gene prediction analysis and manual functional annotation was performed within the Integral Microbial GenomicsExpert Review (IMG-ER) [41] platform developed by the Joint Genome Institute, Walnut Creek, CA, USA.

\section{Genome properties}

The genome includes a circular chromosome of 5,324,944 bp (55\% GC content) along with a circular plasmid of $129,797 \mathrm{bp}$ ( $50 \%$ GC content). The chromosome comprises 5,008 predicted genes while the plasmid comprises 137 predicted genes. 
In total 4,993 genes are assigned as proteincoding genes. About $85 \%$ of the protein-coding genes were assigned to a putative function with the remaining annotated as hypothetical proteins. The genome consists of 22 rRNA genes, 82 tRNA genes and 58 pseudogenes. The properties and the statistics of the genome are summarized in Tables 3 and 4 and Figures $3 a$ and $3 b$.

The genome contains genes arranged in several gene clusters encoding secondary metabolites such as siderophores (enterobactin and aerobactin) and antibiotics (pyrrolnitrin). These compounds can contribute indirectly to plant growth enhancement by suppressing growth of pathogens. The genome also includes genes for the production of plant growth hormones such as indole-3-acetic acid (IAA), which can be directly involved in plant growth. Further studies of the biochemical properties of additional secondary metabolites and regulation of their production using functional genomics will elucidate the detailed mechanisms underlying plant growth promotion by S. proteamaculans S4.

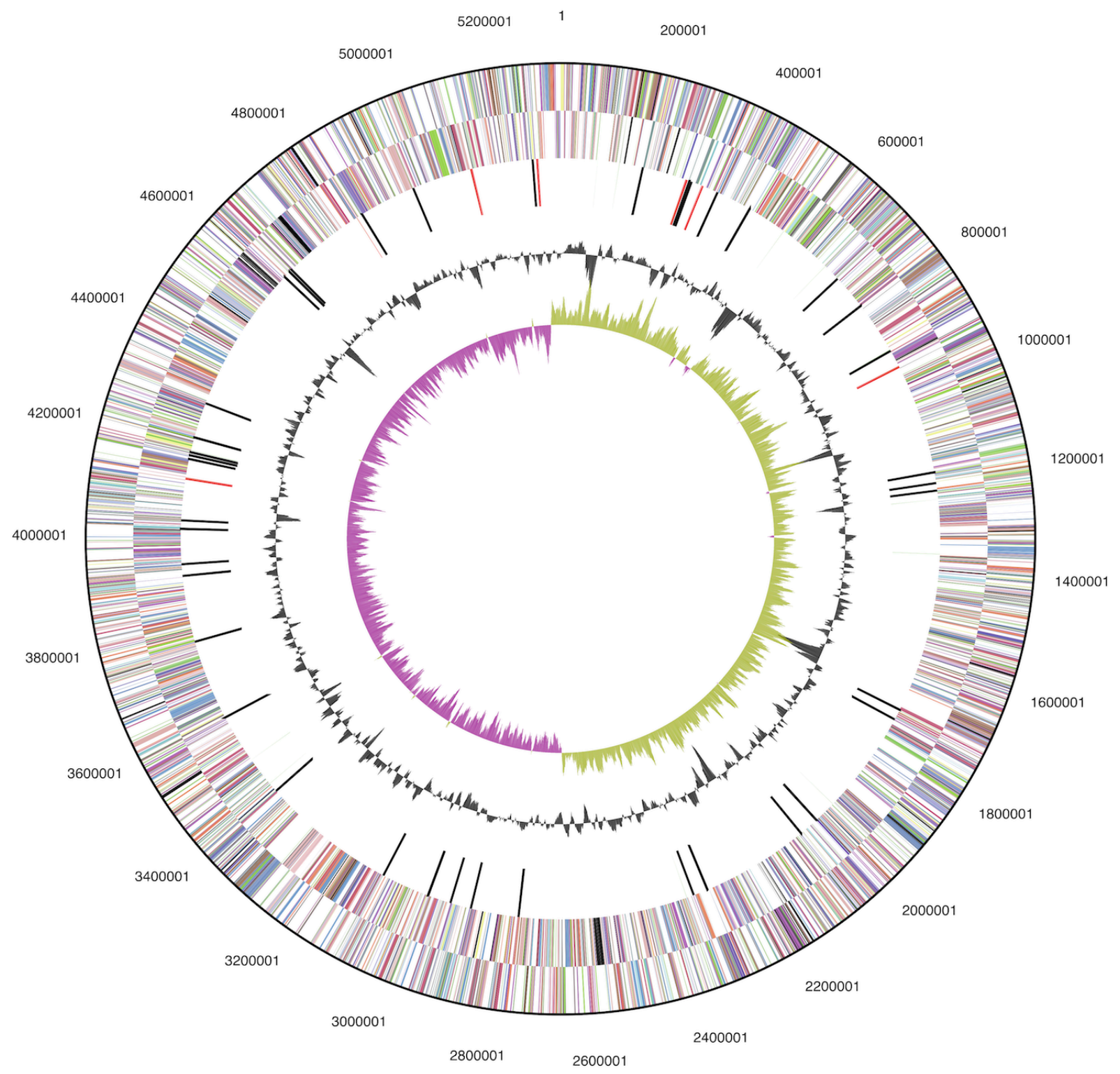

Figure 3a. Graphical circular map of the chromosome. From outside to the center: Genes on forward strand (color by COG categories), Genes on reverse strand (color by COG categories), RNA genes (tRNAs blue, rRNAs red, other RNAs black), GC content, GC skew. 


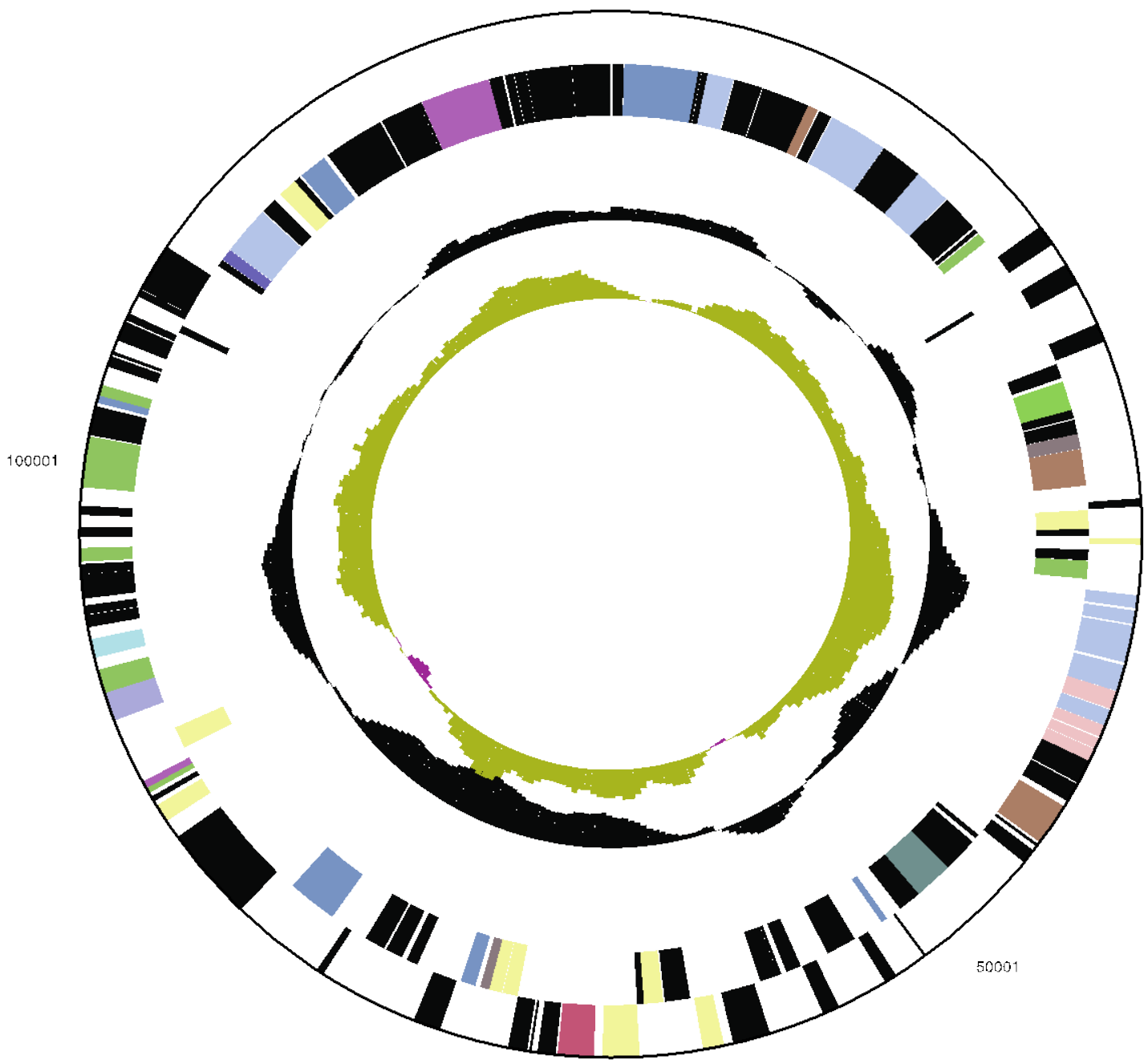

Figure 3b. Graphical circular map of the plasmid. From outside to the center: Genes on forward strand (color by COG categories), Genes on reverse strand (color by COG categories), RNA genes (tRNAs blue, rRNAs red, other RNAs black), GC content, GC skew.

Table 3. Genome statistics

\begin{tabular}{lrr}
\hline Attribute & Value & \% $^{\text {of total }} \mathbf{~}^{\mathbf{a}}$ \\
\hline Genome size (bp) & $5,454,741$ & 100.00 \\
DNA coding region (bp) & $4,825,361$ & 88.46 \\
DNA G+C content (bp) & $2,999,404$ & 54.99 \\
Total genes & 5,142 & 100.00 \\
RNA genes & 149 & 2.90 \\
rRNA operons & 7 & \\
Protein-coding genes & 4,993 & 97.10 \\
Pseudogenes & 58 & 1.13 \\
Genes in paralog clusters & 2,759 & 53.66 \\
Genes assigned to COGs & 4,247 & 82.59 \\
Genes with signal peptides & 1,154 & 22.44 \\
Genes with transmembrane helices & 1,236 & 24.04 \\
\hline
\end{tabular}

a) The total is based on either the size of the genome in base pairs or the total number of protein coding genes in the annotated genome. 
Table 4. Number of genes associated with the 25 general COG functional categories

\begin{tabular}{crrl}
\hline Code & Value & \% of total $^{\text {a }}$ & Description \\
\hline J & 201 & 4.18 & Translation \\
A & 1 & 0.02 & RNA processing and modification \\
K & 452 & 9.41 & Transcription \\
L & 158 & 3.29 & Replication, recombination and repair \\
B & 1 & 0.02 & Chromatin structure and dynamics \\
D & 37 & 0.77 & Cell cycle control, mitosis and meiosis \\
Y & 0 & 0.00 & Nuclear structure \\
V & 57 & 1.19 & Defense mechanisms \\
T & 198 & 4.12 & Signal transduction mechanisms \\
M & 256 & 5.33 & Cell wall/membrane biogenesis \\
N & 142 & 2.96 & Cell motility \\
Z & 0 & 0.00 & Cytoskeleton \\
W & 0 & 0.00 & Extracellular structures \\
U & 166 & 3.46 & Intracellular trafficking and secretion \\
O & 153 & 3.18 & Posttranslational modification, protein turnover, chaperones \\
C & 275 & 5.72 & Energy production and conversion \\
G & 427 & 8.89 & Carbohydrate transport and metabolism \\
E & 487 & 10.14 & Amino acid transport and metabolism \\
F & 109 & 2.27 & Nucleotide transport and metabolism \\
H & 179 & 3.73 & Coenzyme transport and metabolism \\
I & 139 & 2.89 & Lipid transport and metabolism \\
P & 287 & 5.97 & Inorganic ion transport and metabolism \\
Q & 122 & 2.54 & Secondary metabolite biosynthesis, transport and catabolism \\
R & 549 & 11.43 & General function prediction only \\
S & 408 & 8.49 & Function unknown \\
- & 895 & 17.41 & Not in COGs \\
\hline & & & \\
\hline
\end{tabular}

a) The total is based on the total number of protein coding genes in the annotated genome.

\section{Acknowledgements}

The work conducted by the US Department of Energy Joint Genome Institute is supported by the Office of

\section{References}

1. Grimont PA, Grimont F. The genus Serratia. Annu Rev Microbiol 1978; 32:221-248. PubMed http://dx.doi.org/10.1146/annurev.mi.32.100178. $\underline{001253}$

2. Grimont PD, Grimont F, Starr M. Serratia species isolated from plants. Curr Microbiol 1981; 5:317322. http://dx.doi.org/10.1007/BF01567926

3. Kalbe C, Marten P, Berg G. Strains of the genus Serratia as beneficial rhizobacteria of oilseed rape
Science of the U.S. Department of Energy under Contract No. DE-AC02-05CH11231.

with. Microbiol Res 1996; 151:433-439. PubMed http://dx.doi.org/10.1016/S0944-5013(96)80014$\underline{0}$

4. Kurze S, Bahl H, Dahl R, Berg G. Biological control of fungal strawberry diseases by Serratia plymuthica HRO-C48. Plant Dis 2001; 85:529534.

http://dx.doi.org/10.1094/PDIS.2001.85.5.529 
5. Altschul SF, Madden TL, Schäffer AA, Zhang J, Zhang Z, Miller W, Lipman DJ. Gapped BLAST and PSI-BLAST: a new generation of protein database search programs. Nucleic Acids Res 1997; 25:3389-3402. PubMed http://dx.doi.org/10.1093/nar/25.17.3389

6. Larkin MA, Blackshields G, Brown NP, Chenna R, McGettigan PA, McWilliam H, Valentin F, Wallace IM, Wilm A, Lopez R, et al. Clustal W and Clustal X version 2.0. Bioinformatics 2007; 23:2947-2948. PubMed http://dx.doi.org/10.1093/bioinformatics/btm404

7. Tamura K, Peterson D, Peterson N, Stecher G, Nei M, Kumar S. MEGA5: molecular evolutionary genetics analysis using maximum likelihood, evolutionary distance, and maximum parsimony methods. Mol Biol Evol 2011; 28:2731-2739. PubMed http://dx.doi.org/10.1093/molbev/msr121

8. Pattengale N, Alipour M, Bininda-Emonds OP, Moret BE, Stamatakis A. How many bootstrap replicates are necessary? Lect Notes Comput Sci 2009; 5541:184-200.

http://dx.doi.org/10.1007/978-3-642-02008-7_13

9. Liolios K, Chen IMA, Mavromatis K, Tavernarakis N, Hugenholtz P, Markowitz VM, Kyrpides NC. The Genomes On Line Database (GOLD) in 2009: status of genomic and metagenomic projects and their associated metadata. Nucleic Acids Res 2010; 38:D346-D354. PubMed http://dx.doi.org/10.1093/nar/gkp848

10. Auch AF, von Jan M, Klenk HP, Göker M. Digital DNA-DNA hybridization for microbial species delineation by means of genome-to-genome sequence comparison. Stand Genomic Sci 2010; 2:117-134. PubMed http://dx.doi.org/10.4056/sigs.531120

11. Auch AF, Klenk HP, Göker M. Standard operating procedure for calculating genome-to-genome distances based on high-scoring segment pairs. Stand Genomic Sci 2010; 2:142-148. PubMed http://dx.doi.org/10.4056/sigs.541628

12. Field D, Garrity G, Gray T, Morrison N, Selengut J, Sterk P, Tatusova T, Thomson N, Allen MJ, Angiuoli SV, et al. The minimum information about a genome sequence (MIGS) specification. Nat Biotechnol 2008; 26:541-547. PubMed http://dx.doi.org/10.1038/nbt1360

13. Woese CR, Kandler O, Wheelis ML. Towards a natural system of organisms: proposal for the domains Archaea, Bacteria, and Eucarya. Proc Natl
Acad Sci USA 1990; 87:4576-4579. PubMed http://dx.doi.org/10.1073/pnas.87.12.4576

14. Garrity GM, Bell JA, Liburn T. Phylum XIV. Proteobacteria phy nov. In: Garrity GM, Brenner DJ, Krieg NR, Staley JT (eds), Bergey's Manual of Systematic Bacteriology, Second Edition, Volume 2, Part B, Springer, New York, 2005, p. 1.

15. Garrity GM, Bell JA, Liburn T. Class III. Gammaproteobacteria class nov. In: Garrity GM, Brenner DJ, Krieg NR, Staley JT (eds), Bergey's Manual of Systematic Bacteriology, Second edition, Volume 2, Part B. New York: Springer; 2005. p 1.

16. Validation of publication of new names and new combinations previously effectively published outside the IJSEM. Int I Syst Evol Microbiol 2005; 55:2235-2238.

http://dx.doi.org/10.1099/ijs.0.64108-0

17. Garrity GM, Holt JG. Taxonomic Outline of the Archaea and Bacteria. In: Garrity GM, Boone DR, Castenholtz RW (eds), Bergey's Manual of Systematic Bacteriology, Second Edition, Volume 1, Springer, New York, 2001, p. 155-166.

18. Skerman VBD, McGowan V, Sneath PHA. Approved lists of bacterial names. Int I Syst Bacteriol 1980; 30:225-420.

http://dx.doi.org/10.1099/00207713-30-1-225

19. Rahn O. New principles for the classification of bacteria. Zentralbl Bakteriol Parasitenkd Infektionskr Hyg 1937; 96:273-286.

20. Judicial Commission. Conservation of the family name Enterobacteriaceae, of the name of the type genus, and designation of the type species OPINION NO. 15. Int Bull Bacteriol Nomencl Taxon 1958; 8:73-74.

21. Sakazaki R. Genus IX. Serratia Bizio 1823, 288. In: Buchanan RE, Gibbons NE (eds), Bergey's Manual of Determinative Bacteriology, Eighth Edition, The Williams and Wilkins Co., Baltimore, 1974, p. 326.

22. Bizio B. Lettera di Bartolomeo Bizio al chiarissimo canonico Angelo Bellani sopra il fenomeno della polenta porporina. Biblioteca Italiana o sia Giornale di Letteratura. [Anno VIII]. Scienze e Arti 1823; 30:275-295.

23. Grimont PAD, Grimont F, Starr MP. Serratia proteamaculans (Paine and Stansfield) comb. nov., a senior subjective synonym of Serratia liquefaciens (Grimes and Hennerty) Bascomb et al. Int J Syst Bacteriol 1978; 28:503-510. http://dx.doi.org/10.1099/00207713-28-4-503 
24. BAuA. 2010, Classification of bacteria and archaea in risk groups. http://www.baua.de TRBA 466, p. 200.

25. Ashburner M, Ball CA, Blake JA, Botstein D, Butler $\mathrm{H}$, Cherry JM, Davis AP, Dolinski K, Dwight SS, Eppig JT, et al. Gene ontology: tool for the unification of biology. The Gene Ontology Consortium. Nat Genet 2000; 25:25-29. PubMed http://dx.doi.org/10.1038/75556

26. DOE Joint Genome Institute. http://www.jgi.doe.gov.

27. Zerbino DR, Birney E. Velvet: Algorithms for de novo short read assembly using de Bruijn graphs. Genome Res 2008; 18:821-829. PubMed http://dx.doi.org/10.1101/gr.074492.107

28. Ewing B, Hillier L, Wendl MC, Green P. Basecalling of automated sequencer traces using Phred. I. accuracy assessment. Genome Res 1998; 8:175-185. PubMed http://dx.doi.org/10.1101/gr.8.3.175

29. Ewing B, Green P. Base-calling of automated sequencer traces using Phred. II. error probabilities. Genome Res 1998; 8:175-185. PubMed http://dx.doi.org/10.1101/gr.8.3.175

30. Gordon D, Abajian C, Green P. Consed: a graphical tool for sequence finishing. Genome Res

1998; 8:195-202. PubMed http://dx.doi.org/10.1101/gr.8.3.195

31. Lapidus A, LaButti K, Foster B, Lowry S, Trong SEG. POLISHER: An effective tool for using ultra short reads in microbial genome assembly and finishing. AGBT, Marco Island, FL, 2008.

32. Han C, Chain P. Finishing repeat regions automatically with Dupfinisher. In: Proceeding of the 2006 international conference on bioinformatics \& computational biology. Arabina HR, Valafar $\mathrm{H}$ (eds), CSREA Press. June 26-29, 2006: 141-146.

33. Hyatt D, Chen GL, LoCascio P, Land M, Larimer F, Hauser L. Prodigal: prokaryotic gene recognition and translation initiation site identification. BMC Bioinformatics 2010; 11:119. PubMed http://dx.doi.org/10.1186/1471-2105-11-119
34. Mavromatis K, Ivanova N, Chen A, Szeto E, Markowitz V, Kyrpides NC. Standard operating procedure for the annotations of microbial genomes by the production genomic facility of the DOE JGI. Stand Genomic Sci 2009; 1:63-67. PubMed http://dx.doi.org/10.4056/sigs.632

35. Pati A, Ivanova NN, Mikhailova N, Ovchinnikova G, Hooper SD, Lykidis A, Kyrpides NC.

GenePRIMP: a gene prediction improvement pipeline for prokaryotic genomes. Nat Methods 2010; 7:455-457. PubMed http://dx.doi.org/10.1038/nmeth.1457

36. Lowe TM, Eddy SR. tRNAscan-SE: a program for improved detection of transfer RNA genes in genomic sequence. Nucleic Acids Res 1997; 25:955-964. PubMed

37. Lagesen K, Hallin P, Rødland EA, Stærfeldt HH, Rognes T, Ussery DW. RNAmmer: consistent and rapid annotation of ribosomal RNA genes. Nucleic Acids Res 2007; 35:3100-3108. PubMed http://dx.doi.org/10.1093/nar/gkm160

38. Griffiths-Jones S, Bateman A, Marshall M, Khanna A, Eddy SR. Rfam: an RNA family database. Nucleic Acids Res 2003; 31:439-441. PubMed http://dx.doi.org/10.1093/nar/gkg006

39. Krogh A, Larsson B, von Heijne G, Sonnhammer ELL. Predicting transmembrane protein topology with a hidden markov model: application to complete genomes. J Mol Biol 2001; 305:567580. PubMed http://dx.doi.org/10.1006/jmbi.2000.4315

40. Bendtsen JD, Nielsen H, von Heijne G, Brunak S. Improved prediction of signal peptides: SignalP 3.0. J Mol Biol 2004; 340:783-795. PubMed http://dx.doi.org/10.1016/j.jmb.2004.05.028

41. Markowitz VM, Mavromatis K, Ivanova NN, Chen IMA, Chu K, Kyrpides NC. IMG ER: a system for microbial genome annotation expert review and curation. Bioinformatics 2009; 25:2271-2278. PubMed http://dx.doi.org/10.1093/bioinformatics/btp393 